\title{
New Solution of a Spherically Symmetric Static Problem of General Relativity
}

\author{
Valery Vasiliev ${ }^{1}$ \\ ${ }^{1}$ Institute of Problems in Mechanics, Russian Academy of Sciences, 101 Vernadskogo, Moscow 119526, Russia \\ Correspondence: Valery Vasiliev, Institute of Problems in Mechanics, Russian Academy of Sciences, 101 \\ Vernadskogo, Moscow 119526, Russia. Tel: 7-916-607-1281. E-mail: vvvas@dol.ru
}

Received: July 12, 2017

doi:10.5539/apr.v9n5p29
Accepted: July 27, 2017

Online Published: August 21, 2017

\begin{abstract}
The paper is concerned with the spherically symmetric static problem of the General Relativity Theory. The classical solution of this problem found in 1916 by K. Schwarzschild for a particular metric form results in singular space metric coefficient and provides the basis of the objects referred to as Black Holes. A more general metric form applied in the paper allows us to obtain the solution which is not singular. The critical radius of the fluid sphere, following from this solution does not coincide with the traditional gravitational radius. For the spheres with radii that are less than the critical value, the solution of GRT problem does not exist.
\end{abstract}

Keywords: general relativity theory, spherically symmetric problem

\section{Introduction. General Relativity Equations}

General Relativity Theory (GRT) is based on the Einstein equations

$$
\chi T_{i}^{k}=R_{i}^{k}-\frac{1}{2} \delta_{i}^{k} R
$$

which link the energy tensor $T_{i}^{k}$ with the curvature tensor $R_{i}^{k} \quad\left(R=R_{i}^{i}\right)$ that is expressed in terms of the metric tensor of the Riemannian space. Equations (1) include the GRT gravitational constant

$$
\chi=8 \pi G / c^{4}
$$

which depends on the classical gravitational constant $G$ and the velocity of light $c$. The energy tensor $T_{i}^{k}$ expressed with the aid of Equations (1) identically satisfies the conservation equations

$$
T_{i, k}^{k}=0
$$

We use mixed tensor components because for the spherically symmetric problem considered below they coincide with the physical components.

For four-dimensional space-time, we have ten Equations (1) for the same number of the metric tensor coefficients. However, since the left-hand parts of Equations (1) identically satisfy four Equations (3), only six Equations (1) are mutually independent. Thus, if the tensor $T_{i}^{k}$ is known, we have six equations for ten unknown functions, and the system of GRT equations is not complete.

\section{Equations of Spherically Symmetric Problem}

For spherically symmetric static problem, the basic metric form can be presented as

$$
d s^{2}=g^{2} d r^{2}+\rho^{2}\left(d \theta^{2}+\sin ^{2} \theta d \phi^{2}\right)-h^{2} c^{2} d t^{2}
$$

in which $r, \theta, \phi$ and $t$ are space spherical and time coordinates, $g, \rho, h$ are the metric coefficients that depend on the radial coordinate $r$ only. For the spherically symmetric static problem, the energy tensor is

$$
T_{1}^{1}=\sigma_{r}, T_{2}^{2}=T_{3}^{3}=\sigma_{\theta}, T_{4}^{4}=\mu c^{2}
$$


where $\sigma_{r}$ and $\sigma_{\theta}$ are the radial and the circumferential stresses and $\mu$ is the space density. In accordance with the closure of the Introduction, the system of Equations (1) is not complete and for three metric coefficients $g, \rho, h$ we have two mutually independent equations (1), i.e.

$$
\frac{1}{\rho^{2}}-\frac{\rho^{\prime}}{\rho g^{2}}\left(\frac{\rho^{\prime}}{\rho}+\frac{2 h^{\prime}}{h}\right)=\chi \sigma_{r}, \frac{1}{\rho^{2}}-\frac{1}{g^{2}}\left[\left(\frac{\rho^{\prime}}{\rho}\right)^{2}-\frac{2}{\rho}\left(\rho^{\prime \prime}-\frac{\rho^{\prime} g^{\prime}}{g}\right)\right]=\chi \mu c^{2}
$$

Here, $(\ldots)^{\prime}=d(\ldots) / d r$. For the spherically symmetric static problem, Equation (3) reduces to

$$
\sigma_{r}^{\prime}-\frac{2 \rho^{\prime}}{\rho}\left(\sigma_{\theta}-\sigma_{r}\right)+\frac{h^{\prime}}{h}\left(\sigma_{r}-\mu c^{2}\right)=0
$$

Thus, we have three equations, Equations (6) and (7), for six unknown functions - three metric coefficients and three stresses. Equation (7) is actually the equilibrium equation for a solid sphere (Vasiliev \& Fedorov, 2014). As follows from the Theory of Elasticity, to find the stresses, Equation (7) must be supplemented with constitutive equations linking stresses and strains and compatibility equations for the strains. Such equations do not exist in GRT and it cannot, in general, be applied to study the gravitation in deformable solids. There are only two particular cases for which the problem can be solved. The first case corresponds to the empty space in which stresses and density are zero and Equation (7) does not exist. In this case, we have two Equations (6) for three functions $g, \rho, h$ and the system is not complete. The second case corresponds to a sphere consisting of a perfect fluid for which $\sigma_{r}=\sigma_{\theta}=-p$, where $p$ is the pressure that can be found from Equation (7) which takes the form

$$
p^{\prime}+\frac{h^{\prime}}{h}\left(p+\mu c^{2}\right)=0
$$

The system of three equations, Equations (6) and (8) includes four unknown functions $g, \rho, h, p$ and again is not complete. To solve the problem, the coordinate system can be specified in various ways (Synge, 1960) which actually means that one of the metric coefficients can be preassigned. Possible forms of solution for spherically symmetric static problem are discussed by Vasiliev \& Fedorov (2015). One more solution which is a direct generalization of the classical Schwarzchild solution is presented below.

\section{The Schwarzchild Solution}

Spherically symmetric GRT problem for a fluid sphere was solved by K. Schwarzchild in 1916. Following Synge (1960), this solution is discussed briefly in this Section. The complete set of equations formed by Equations (6) and (8) was obtained under the condition $\rho(r)=r$. Then, Equations (6) reduce to

$$
\frac{1}{r g^{2}}\left(\frac{1}{r}+\frac{2 h^{\prime}}{h}\right)-\frac{1}{r^{2}}=\chi p, \quad \frac{1}{r^{2}} \frac{d}{d r}\left(r-\frac{r}{g^{2}}\right)=\chi \mu c^{2}
$$

Consider a fluid sphere with radius $R$. For the external empty space $(r \geq R)$, we should take $p=0$ and $\mu=0$. The solution of Equations (9) is well known and has the form

$$
g_{e}^{2}=\frac{1}{1-r_{g} / r}, \quad h_{e}^{2}=1-\frac{r_{g}}{r}
$$

where index " $e "$ corresponds to the external space and

$$
r_{g}=2 m G / c^{2}
$$

is the so-called gravitational radius in which $m$ is the sphere mass. For the internal space $(0 \leq r \leq R)$, the solution must satisfy the space symmetry condition at the sphere center according to which

$$
g_{i}(r=0)=1
$$

Index " $i$ " corresponds to the internal space. For the sphere with constant density $\mu$, the solution of the second equation of Equations (9) which satisfies Equation (12) is

$$
g_{i}^{2}=\frac{1}{1-u r^{2} / R^{2}}
$$


where

$$
u=\frac{1}{3} \chi \mu c^{2} R^{2}
$$

is a dimensionless parameter. The solutions in Equations (10) and (13) must satisfy the boundary condition on the sphere surface according to which

Applying this condition, we get

$$
g_{e}(r=R)=g_{i}(r=R)=g_{R}
$$

$$
u=r_{g} / R
$$

and Equation (13) can be reduced to the final form

$$
g_{i}^{2}=\frac{1}{1-r_{g} r^{2} / R^{3}}
$$

To complete the solution, we need to determine the pressure in the fluid. Substituting Equation (17) in the first equation of Equations (9) and expressing $h$, we get

$$
\frac{h^{\prime}}{h}=\frac{1}{2}\left[\frac{r}{1-r_{g} r^{2} / R^{3}}\left(\frac{1}{r^{2}}+\chi p\right)-\frac{1}{r}\right]
$$

Substituting further Equation (18) in Equation (8) and using Equation (2) for $\chi$, we arrive at the following equation for the pressure:

$$
p^{\prime}+\frac{r_{g} r}{2\left(R^{3}-r_{g} r^{2}\right)}\left(p+\mu c^{2}\right)\left(1+\frac{3 p}{\mu c^{2}}\right)=0
$$

The solution of this equation which satisfies the boundary condition on the sphere surface

$$
p(r=R)=0
$$

is

$$
p(r)=-\mu c^{2} \frac{\sqrt{1-r_{g} r^{2} / R^{3}}-\sqrt{1-r_{g} / R}}{\sqrt{1-r_{g} r^{2} / R^{3}}-3 \sqrt{1-r_{g} / R}}
$$

The Schwarzchild solution for a fluid sphere is analyzed by Vasiliev and Fedorov (2014). The brief results of this analysis are.

1. As follows from Equations (10) and (17), the radial metric coefficient $g$ is singular on the sphere surface $r=R$ if the radius takes the critical value $R=r_{g}$.

2. Substituting $u$ from Equation (14) in Equation (16) and using Equations (2) and (11) for $\chi$ and $r_{g}$, we can find the sphere mass which is

$$
m=m_{0}=\frac{4}{3} \pi \mu R^{3}
$$

This expression corresponds to the Euclidean space. However, the space inside the sphere is Riemannian. The metric coefficient of this space is specified by Equation (17) and the mass should be

$$
m=4 \pi \mu \int_{0}^{R} g_{i} r^{2} d r=4 \pi \mu \int_{0}^{R} \frac{r^{2} d r}{\sqrt{1-r_{g} r^{2} / R^{3}}}=\frac{3 m_{0} R}{2 r_{g}}\left(\sqrt{\frac{R}{r_{g}}} \sin ^{-1} \sqrt{\frac{r_{g}}{R}}-\sqrt{1-\frac{r_{g}}{R}}\right)
$$

Putting, for example $R=r_{g}$, we have $m=3 \pi m_{0} / 4$. Taking the sphere mass in accordance with Equation (23) and ignoring Equation (22), we cannot satisfy the boundary condition in Equation (15).

3. Taking $r=0$ in Equation (21) find the pressure at the sphere center, i.e. 


$$
p_{0}=\mu c^{2} \frac{1-\sqrt{1-r_{g} / R}}{1-3 \sqrt{1-r_{g} / R}}
$$

The denominator of this expression is zero for the sphere with radius $R=R_{g}=9 / 8 r_{g}=1.125 r_{g}$, and the pressure becomes infinitely high at the sphere center. Thus, the admissible radius for the Schwarzchild solution is $R_{g}$. The metric coefficients in Equations (10) and (17) are not singular on the sphere surface if $R=R_{g}$.

The GRT equations for a fluid sphere analogous to Equations (8) and (9) have been studied by various authors. Particularly, Tolman (1939) and Adler (1974) assuming that three equations include four unknown functions, i.e. $g(r), h(r)$ and $p(r), \mu(r)$, and preassigning the metric coefficients $g$ or $h$, have found a set of particular solutions determining pressure and density. More realistic approach has been demonstrated by Oppenheimer and Volkoff (1939) who linked pressure and density by some constitutive equation and obtained the numerical solution. Buchdahl (1959) has performed qualitative analysis of the equations and established limitations under which the solution can exist.

It should be emphasized that the aforementioned results correspond to the Schwarzchild form of the line element specified by Equation (4) in which $\rho(r)=r$. In this paper, a more general form of the function $\rho(r)$ is applied.

\section{Generalized Solution}

Taking into account the foregoing specific features of the Schwarzchild solution return to the metric form in Equation (4) and assume that $\rho(r)$ is some unknown function. Since the metric coefficient $g$ is different for the external ( $r \geq R$, index " $e$ ") and internal ( $0 \leq r \leq R$, index " $i$ ") spaces in the Schwarzchild solution, we can assume that the functions $\rho_{e}(r)$ and $\rho_{i}(r)$ are also different. Naturally, these functions must satisfy some conditions. Particularly, we require that

$$
\rho_{e}(r \rightarrow \infty) \rightarrow r, \quad \rho_{i}(r=0)=0, \quad \rho_{e}(r=R)=\rho_{i}(r=R)=\rho_{R}
$$

Consider the second equation of Equations (6) which can be reduced to the following form:

$$
1-\frac{1}{\rho^{\prime}} \frac{d}{d r}\left[\frac{\rho}{g^{2}}\left(\rho^{\prime}\right)^{2}\right]=\chi \mu c^{2} \rho^{2}
$$

For the external $(r \geq R)$ space, we should take $\mu=0$. Since the solution must satisfy the first condition in Equations (24), it must reduce to the solution in Equations (10) for $\rho_{e} \rightarrow \infty$. Finally, the solution of Equation (25) becomes

$$
g_{e}^{2}=\frac{\left(\rho_{e}^{\prime}\right)^{2}}{1-r_{g} / \rho_{e}}
$$

Substituting this result in the first equation of Equations (6), taking $\sigma_{r}=0$ and integrating, we get

$$
h_{e}^{2}=1-\frac{r_{g}}{\rho_{e}}
$$

For $\rho(r)=r$, Equations (26) and (27) degenerate in to Equations (10) corresponding to the Schwarzchild solution.

For the internal $(R \geq r \geq 0)$ space, the solution of Equation (25) is

$$
g_{i}^{2}=\frac{\left(\rho_{i}^{\prime}\right)^{2}}{1-u \rho_{i}^{2} / R^{2}}
$$

Here, $u$ is specified by Equation (14). To satisfy the space symmetry condition at the sphere center in Equation (13), we should impose the condition on $\rho_{i}$ requiring that

$$
\rho_{i}^{\prime}(r=0)=1
$$

Perform some transformations. Substituting $\chi$ from Equation (2) in Equation (14) and using Equations (11) and (22) for $r_{g}$ and $m_{0}$, we arrive at

$$
u=\frac{r_{g} m_{0}}{R m}=\bar{r}_{g} \frac{m_{0}}{m}, \quad \bar{r}_{g}=\frac{r_{g}}{R}
$$

If $m=m_{0}$, Equation (30) reduces to Equation (16) corresponding to the Schwarzchild solution. However in general, the sphere mass is expressed as 


$$
\begin{gathered}
m=4 \pi \mu \int_{0}^{R} g_{i} \rho_{i}^{2} d r=4 \pi \mu \int_{0}^{R} \frac{\rho_{i}^{\prime} \rho_{i}^{2} d r}{\sqrt{1-u \rho_{i}^{2} / R^{2}}}=4 \pi \mu \int_{0}^{\rho_{R}} \frac{\rho_{i}^{2} d \rho_{i}}{\sqrt{1-u \rho_{i}^{2} / R^{2}}}=4 \pi \mu R^{3} \int_{0}^{\bar{\rho}_{R}} \frac{\bar{\rho}_{i}^{2} d \bar{\rho}_{i}}{\sqrt{1-u \bar{\rho}_{i}^{2}}} \\
=\frac{3 m_{0}}{2 u}\left[\frac{1}{\sqrt{u}} \sin ^{-1}\left(\bar{\rho}_{R} \sqrt{u)}-\bar{\rho}_{R} \sqrt{1-u \bar{\rho}_{R}^{2}}\right]\right.
\end{gathered}
$$

where

$$
\bar{\rho}=\frac{\rho}{R}, \quad \bar{\rho}_{R}=\frac{\rho_{R}}{R}
$$

and $\rho_{R}$ is specified by Equation (24).

As follows from Equations (23) and (31), the sphere mass depends on gravitation (Zeldovich \& Novikov, 1971). If this is the case, Equations (30) and (31) yield

$$
\bar{r}_{g}=\frac{3}{2}\left[\frac{1}{\sqrt{u}} \sin ^{-1}\left(\bar{\rho}_{R} \sqrt{u}\right)-\bar{\rho}_{R} \sqrt{1-\bar{\rho}_{R}^{2} u}\right]
$$

To solve the problem, we need to specify the functions $\rho_{e}(r)$ and $\rho_{i}(r)$. The simplest functions that satisfy the conditions in Equations (12) and (24) are

$$
\bar{\rho}_{e}=\bar{r}\left(1+\frac{e}{\bar{r}}\right), \quad \bar{\rho}_{i}=\bar{r}(1+e \bar{r}), \quad \bar{r}=\frac{r}{R}
$$

Here, $e$ is some unknown parameter depending on the intensity of gravitation. Substituting Equations (34) in Equations (26) and (28), we get

$$
g_{e}^{2}=\frac{1}{1-\bar{r}_{g} /(\bar{r}+e)}, \quad g_{i}^{2}=\frac{1+2 e \bar{r}}{1-u \bar{r}^{2}(1+e \bar{r})^{2}}
$$

Parameter $e$ can be found from the boundary condition in Equation (15) which yields

$$
(1+e)^{3} u-(1+2 e)^{2} \bar{r}_{g}+4 e(1+e)^{2}=0
$$

Thus, we have two equations, Equations (33) in which $\bar{\rho}_{R}=1+e$ and Equation (36). These equations include three parameters - $e, u$ and $\bar{r}_{g}$. For the sphere with known radius $R$ and density $\mu$, we can calculate $u$ using Equation (14) and determine $e$ and $\bar{r}_{g}$ applying Equations (33) and (36). Using further Equation (30), we can find the normalized mass $\bar{m}=m / m_{0}$.

To undertake the parametric analysis, we preassign various values of parameter $e$ and find the corresponding parameters $\bar{r}_{g}$ and $u$ (or $\bar{m}$ ) with the aid of Equations (33) and (36). The results of numerical analysis are presented in Figure 1. The solid line corresponds to parameter $e$, whereas the dashed line shows the dependence of the normalized mass $\bar{m}=m / m_{0}$ on the ratio $R / r_{g}$.

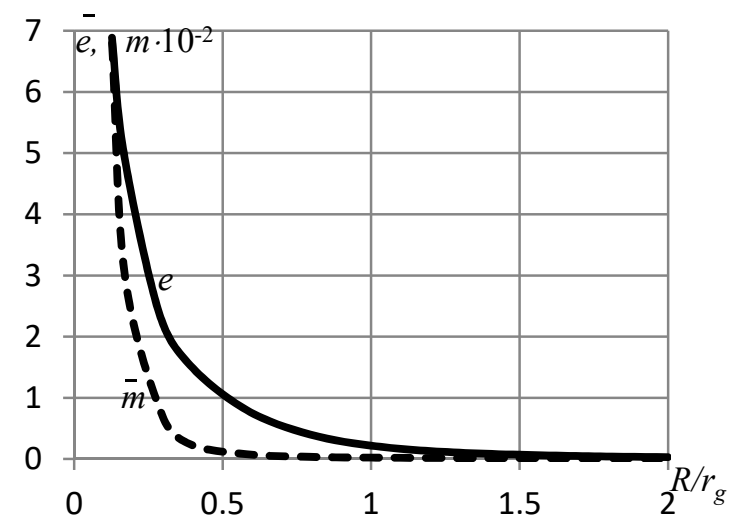

Figure 1. Dependences of parameter $e$ (solid line) and the normalized mass $\bar{m}$ (dashed line) on the ratio $R / r_{g}$ As can be seen, for $R / r_{g}>2$ the parameter $e$ is close to zero, the mass ratio is close to unity and the obtained solution practically coincides with the Schwarzchild solution. The numerical analysis shows that the solution of 
Equations (33) and (36) exists if $R \geq R_{e}=0.127 r_{g}$. For the sphere with the critical radius $R=R_{e}$, the solution is not singular and we have $e=6.887$ and $\bar{m}=680$. For $R<R_{e}$, the solution becomes imaginary.

To proceed, we need to determine the pressure in the fluid. The equation for the pressure analogous to Equation (19) but corresponding to $\rho(r)$ has the following form (Vasiliev \& Fedorov, 2014):

$$
\frac{d p}{d \rho_{i}}+\frac{r_{g} \rho_{i}}{2\left(R^{3}-r_{g} \rho_{i}^{2}\right)}\left(p+\mu c^{2}\right)\left(1+\frac{3 p}{\mu c^{2}}\right)=0
$$

This equation coincides with Equation (19) if we change $\rho_{i}$ to $r$. The solution of Equation (37) which satisfies the boundary condition on the sphere surface $p\left(\rho_{i}=\rho_{R}\right)=0$ is similar to Equation (21), i.e.

$$
p\left(\rho_{i}\right)=-\mu c^{2} \frac{\sqrt{1-r_{g} \rho_{i}^{2} / R^{3}}-\sqrt{1-r_{g} \rho_{R}^{2} / R^{3}}}{\sqrt{1-r_{g} \rho_{i}^{2} / R^{3}}-3 \sqrt{1-r_{g} \rho_{R}^{2} / R^{3}}}
$$

Taking $\rho_{i}=0$, we have for the pressure at the sphere center

$$
p_{0}=\mu c^{2} \frac{1-\sqrt{1-\bar{r}_{g} \bar{\rho}_{R}^{2}}}{1-3 \sqrt{1-\bar{r}_{g} \rho_{R}^{2}}}
$$

where $\rho_{R}=1+e$. The denominator in Equation (38) becomes zero if

$$
\bar{r}_{g}(1+e)^{2}=8 / 9
$$

Using the results of numerical solution (see Figure 1), we get $e=e_{g}=0.0954, R=R_{g}^{e}=1.3388 r_{g}$. Thus, for $R=R_{g}^{e}$ the pressure at the sphere center becomes infinitely high. The obtained admissible radius is higher than the radius following from the Schwarzchild solution $\left(R_{g}=1.125 r_{g}\right)$.

Dependences of $g(\bar{r})$ and $\bar{\rho}(\bar{r})$ for the sphere with the radius $R=R_{g}^{e}$ are presented in Figure 2 with solid lines. Dashed lines correspond to the Schwarzchild solution for the sphere with radius $R=R_{g}$.

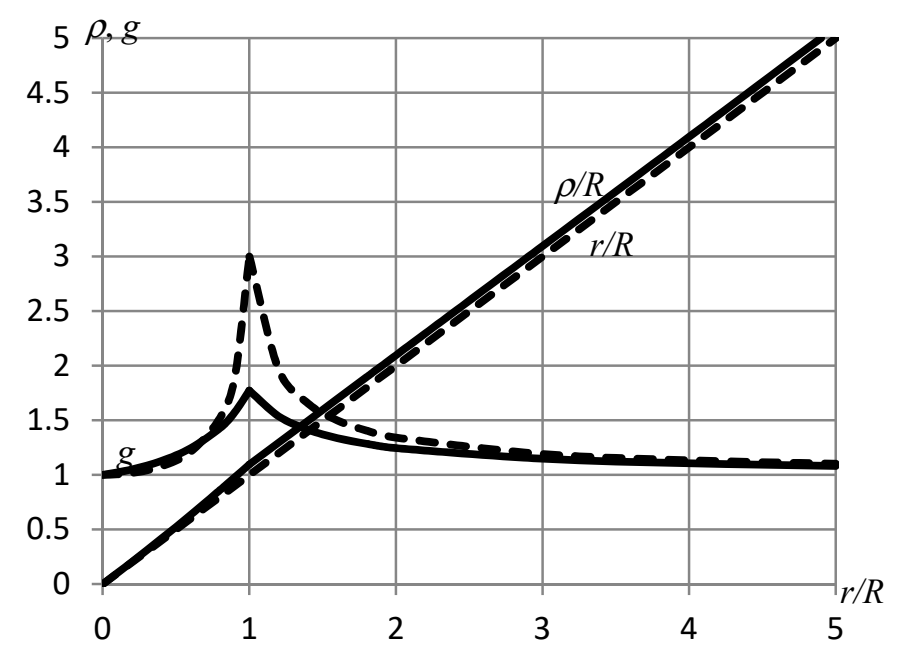

Figure 2. Dependences of the metric coefficients on the radial coordinate

- the obtained solution for $R=R_{g}^{e}$

- the Schwarzchild solution for $R=R_{g}$ 


\section{Conclusion}

The solution of the static spherically symmetric static problem of GRT for a fluid sphere is obtained under the proposed approximation of the circumferential metric coefficient of the line element. The solution provides the critical radius which is different from the classical Schwarzchild radius. For the spheres whose radius is less than the critical value, the solution becomes imaginary. The admissible value of the sphere radius in both Schwarzchild and obtained solutions is restricted by the pressure which becomes infinitely high at the sphere center.

\section{References}

Adler, R. J. (1974). A fluid sphere in general relativity. Journal of Mathematical Physics, 15, 727-729, http://dx.doi.org/10.1063/1.1666717

Buchdahl, H. A. (1959). General relativistic fluid spheres. Physical Review, 116(4), 1027-1035.

Oppengeimer, J. R., \& Volkoff, G. M. (1939). On massive neutron cores. Physical Review, 55, 374-388.

Synge, J. L. (1960). Relativity: the General Theory. Amsterdam, North Holland.

Tolman, R. C. (1939). Static solutions of Einstein's field equations for spheres of fluid. Physical Review, 55, 364373.

Vasiliev V. V., \& Fedorov, L. V. (2014). Stressed state of an elastic ball in spherically symmetric gravitation field. Mechanics od Solids, 49(4), 370-381.

Vasiliev V. V., \& Fedorov, L. V. (2015). On the solution of the spherically symmetric static problem for a fluid sphere in general relativity. Applied Physics Research, 6(3), 40-49. http://dx.doi.org/10.5539/apr.v6n3p40

Vasiliev V. V., \& Fedorov, L. V. (2015). Possible forms of the solution for spherically symmetric static problem in general relativity. Applied Physics Research, 7(3), 10-17. http://dx.doi.org/10.5539/apr.v7n3p10

Zeldovich, Y. B., \& Novikov, I. D. (1971). Theory of Gravitation and Evolutions of Stars. Moscow, Nauka (in Russian).

\section{Copyrights}

Copyright for this article is retained by the author(s), with first publication rights granted to the journal.

This is an open-access article distributed under the terms and conditions of the Creative Commons Attribution license (http://creativecommons.org/licenses/by/4.0/). 